\title{
Scheduled Notification Message
}

National Cancer Institute

\section{Source}

National Cancer Institute. Scheduled Notification Message. NCI Thesaurus. Code C94079.

The actual text scheduled to be included in the notification. 